\title{
IRRADIATION OF SPERM TAILS BY LASER MICROBEAM*
}

\author{
BY STUART F. GOLDSTEIN† \\ Division of Biology, California Institute of Technology, Pasadena, \\ California, 9 г г $\circ$, U.S.A.
}

(Received io February 1969)

\section{INTRODUCTION}

There is much evidence that the movements of flagella are generated within the flagellum itself, and depend on the cell body primarily for the supply of an energy substrate, probably ATP (e.g. Gray, 1955; Hoffmann-Berling, 1955; Brokaw, 1961, 1963). However, little understanding has been gained of the function of particular regions of a flagellum, and little is known about the nature and effects of interactions along a flagellum or the ability of small regions to initiate, maintain and propagate waves independently. In the present study an attempt has been made to localize function and examine functional interactions along a flagellum by observing the effects of localized damage from a ruby laser microbeam.

Engelmann ( 1898 ) reported that frog spermatozoa continued to beat if they were severed between the head and mid-piece, but stopped if severed between the midpiece and tail. This suggests that beats can be initiated only in or near the mid-piece, but these effects could have been due to isolation of the tail from the ATP generating system in the mid-piece. However, recent work with ATP-reactivated glycerinated sea-urchin spermatozoa, which presumably are not dependent on the mid-piece for their energy, indicates that the motility of these flagella is inhibited when they are broken beyond the mid-piece by mechanical shearing (Brokaw \& Benedict, I968). Both normal and glycerinated spermatozoa have been observed to beat after the loss of the head and mid-piece (Brokaw, 1965, and unpublished observations), suggesting that a part of the flagellum near the mid-piece, rather than the mid-piece itself, may be required for motility.

Some observations have been made on the very long and relatively complex sperma. tozoa of urodele amphibians. Baker $(1963)$ observed that in tails of Cryptobranchus spermatozoa, which were impeded by other spermatozoa or debris, cessation of beating near the proximal end did not prevent beating of more distal regions of the tail. In fact, any part could be motile while other parts were non-motile. Terni (1933) found that pricking the undulating membrane of spermatozoa of Geotriton fuscus with a fine needle caused beating to stop at the point touched, and to reverse direction on both sides of that point. He claimed that if he cut the tail into two or more pieces each piece was capable of beating. He also irradiated $8 \mu \mathrm{m}$. sections of these $700 \mu \mathrm{m}$. tails with an ultraviolet microbeam. The irradiated region stopped immediately, but beating remained normal on both sides of that region.

- This paper has been abstracted from part of a Ph.D. thesis presented to the California Institute of Technology.

† Present address: Department of Physiology-Anatomy, University of California, Berkeley, California, 94720 , U.S.A. 
Similar results were reported by Walker ( $196 \mathrm{I}$ ), who irradiated short regions of Trypanosoma with a focused beam of visible light, to which the organism had been sensitized by the presence of acriflavine. The irradiated region stopped beating, while the flagellum on both sides of that region continued to beat. Holwill (1955) noted that in a related organism, Strigomonas oncopelti, when the movement of a flagellum was prevented at one point, distally directed waves were observed on the proximal side of the obstruction and proximally directed waves were observed on the distal side. These trypanosomes are normally capable of propogating waves in either direction along the flagellum.

Somewhat conflicting observations have been reported for more typical spermatozoa which propagate waves in only one direction. Gray (1955) reported observing a sea-urchin spermatozoon attached to a slide by a point in the central part of its flagellum. The region of the tail proximal to the constraint continued to beat, while the remainder of the tail did not. As the spermatozoon moved slowly forward, points on the tail beat as they became proximal to the constraint. On the other hand, Nelson $(1959,1962)$ observed tails of rat spermatozoa beating distally to regions that had become attached to a slide. More extensive observations of this type were made by Kaneda (1965) on frog spermatozoa. He found that restricting the amplitude of part of a flagellum by drawing it into a short length of capillary tubing caused a reduction in frequency which was approximately constant all along the tail, while constraining a point on the tail by pressing it to the coverglass caused the parts of the tail on the two sides of the constraint to beat at different frequencies. If the constraint was placed within $20 \%$ of the tail length from the proximal end, the region between the head and the constraint usually did not beat.

It is not clear from the above observations whether beating distal to a constraint indicates the capability for independent activity in isolated regions of a simple flagellum or whether a bending signal can pass through a mechanical constraint. In the present study short regions of actively beating flagella of echinoderm sperm tails have been broken by a single pulse from a ruby laser microbeam. These flagella have a relatively simple ultrastructure, and their components are probably common to most motile cilia and flagella (Afzelius, 1955). The short pulse duration allows study of the results of irradiation on waves which were established before the irradiation, and it is therefore possible to distinguish between the ability of a region of the flagellum to propagate waves and its ability to initiate bending.

\section{METHODS AND MATERIALS}

Spermatozoa were collected from a sea urchin, Strongylocentrotus purpuratus, following injection of $0.5 \mathrm{M}-\mathrm{KCl}$ into the perivisceral cavity (Tyler, I949), and from a starfish, Pisaster brevispinus, following spontaneous spawning. Live spermatozoa were prepared for use by diluting with a solution of filtered sea water containing $10^{-3} \mathrm{M}$ ethylenediaminetetra-acetate (Tyler, 1953 ), $2 \%$ polyvinyl pyrrolidinone and I $\mathrm{mg} . / \mathrm{ml}$. Food, Drug and Cosmetic blue no. I dye. (Colour Index, 1956. Index number 42090. Obtained in $89 \%$ pure form from H. Kohnstamm \& Co., Huntington Park, California. Also available as brilliant blue FCF.) The $\mathrm{pH}$ of the sea water solution was adjusted to $8 . \mathrm{I}$ with $0.5 \mathrm{M}$ tris (hydroxymethyl) aminomethane. The blue dye did not noticeably affect the movement of the spermatozoa, and absorbed enough of the 
$694.3 \mathrm{~nm}$. radiation to produce reliable damage. A small drop of this suspension was placed on the slide and a cover glass was lowered over the top. $S$. purpuratus eggs were added to the sea-urchin sperm suspension, so that a few eggs were in the drop placed on the slide, and burst when the cover glass was lowered. Spermatozoa were selected for study which were attached to the glass by their heads, with their tails beating freely and regularly. A few such spermatozoa could usually be found in the vicinity of each egg. Starfish spermatozoa often attached themselves to the glass in this way, and no eggs were used with them. A few observations were made with glycerinated spermatozoa, prepared by procedures similar to those described by Brokaw (I966a), with $\mathrm{I} \mathrm{mg} . / \mathrm{ml}$. of the blue dye added to the reactivating solution.

A Zeiss GFL microscope with a trinocular head and a $40 \times$ oil-immersion objective was used with dark-field stroboscopic illumination. A Robot Volläutomat star II $35 \mathrm{~mm}$. camera was mounted above the vertical tube of the microscope. Photomicrographs were taken at a magnification of $200 \times$ on Kodak Tri-X or Ilford Mark V films. Negative prints, such as those reproduced in Plate I, were made from intermediate positives on Kodak Contrast Process Ortho film to increase contrast. A TRG model $5^{1} 3$ biolaser system (TRG Incorporated, Route Iro, Melville, N.Y.) was mounted directly on the vertical tube of the microscope. Laser emission was directed down the tube by a partially reflecting mirror between the camera and the monocular eyepiece, and was focused by the microscope to a nominal diameter of about $2 \mu \mathrm{m}$. The laser was aligned by adjusting the mirror so that a hole burned in a thin film of dye with the laser coincided with cross hairs in one of the viewing eyepieces (Saks, Zuzolo \& Kopac, 1965).

Results were recorded with multiple exposures on a single frame (Gray, 1958; Brokaw, 1963, 1965). The system was designed to allow the laser discharge and the photographic flashes to be synchronized with the beating of a sperm tail, so that the flagellum could be hit at a desired point with the microbeam. In previous studies by Brokaw (1963, 1965), illumination was provided by a General Electric FT-23o shortgap xenon flash lamp, operated at up to $2300 \mathrm{~V}$. with external triggering. For the present experiments the same flash lamp was operated at $4000 \mathrm{~V}$., at which it fired without external triggering, and was ignited by connecting across a flash capacitor by means of a Type 5949 hydrogen thyratron. Two thyratrons were connected to the flash lamp. One acted as a switch for a $0.07 \mu \mathrm{F}$. capacitor to provide low-intensity flashes for observation; the other acted as a switch for a $4 \mu \mathrm{F}$. capacitor to provide high-intensity flashes for photography. The photographic flash capacitor was recharged from a $45 \mu \mathrm{F}$. storage capacitor. The intensity of successive exposures diminished as this storage capacitor discharged, providing a means of verifying the order in which the exposures were taken. The thyratrons were triggered by the amplified trigger output pulses of a General Radio type I53I-A Strobotac. Output pulses from the Strobotac were directed to the appropriate thyratron by means of the shutter contacts of the camera. When a spermatozoon was selected for study the frequency of illumination was adjusted to be equal to that of the tail, providing a stationary image of the flagellum, and the desired point on this image was positioned under the cross hairs. The laser was triggered to fire by the first photographic trigger pulse, by means of a silicon-controlled rectifier placed in series with the manual laser trigger switch. There is a delay of a few tenths of a millisecond in the laser discharge 
(Lengyel, I962), so that the first exposure shows the flagellum just before it was irradiated. An external timer circuit was switched into the Strobotac by the first photographic trigger pulse, to produce a predetermined number of photographic flashes at a predetermined frequency. A more complete description of the apparatus is given in Goldstein (1968).

\section{OBSERVATIONS}

The photographs shown in Plate I were taken with the photographic flashes firing at $50 \mathrm{~Hz}$., after the first flash, which was synchronized with the spermatozoon and the laser discharge. They have been selected to illustrate the results discussed in the text from approximately 100 successful photographs of irradiated spermatozoa. No consistent differences were found between the effects of irradiation in the two species. The frequency of beating of a spermatozoon before irradiation was recorded from the Strobotac; other parameters of beating before irradiation were determined from the first exposure on the photograph. This exposure can usually be readily identified as being more intense than the subsequent ones. The damaged point usually appears in the same position on the last few exposures of a photograph, having apparently become attached to the glass. That portion of the flagellum between the head and the irradiated point is referred to as 'proximal'; that portion beyond the damaged point is referred to as 'distal'.

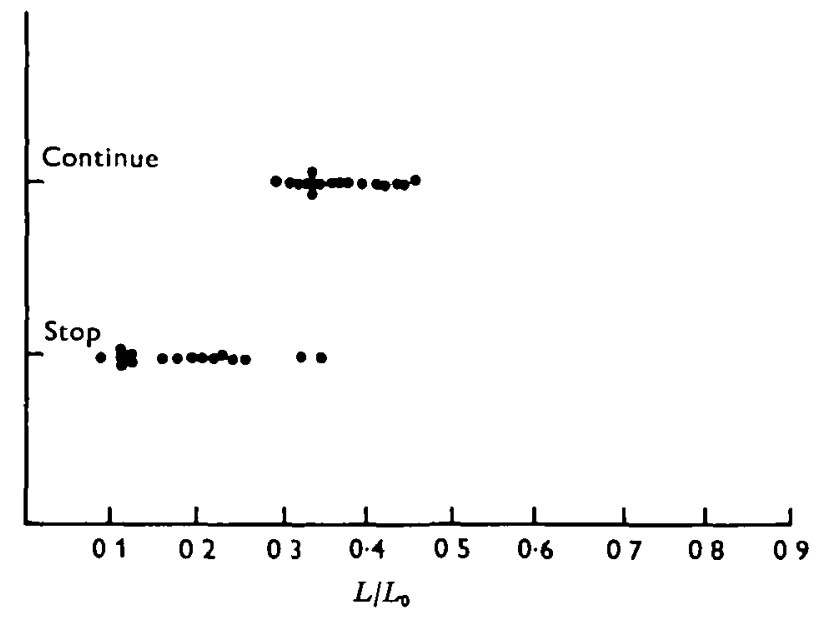

Text-fig. I. Continuation of beating in the proximal portion as a function of 1 ta length. Points indicate individual flagella. $L$, length of proximal portion; $L_{0}$, length of entire tail.

\section{Effects on the proximal region of live spermatozoa}

In the spermatozoa shown in Pl. I, figs. I and 2 the movement of the proximal portion of the flagellum was immediately and permanently inhibited following irradiation. In the spermatozoa shown in Pl. I, figs. $3^{-5}$ the proximal portion continued to beat. There is no indication that the ability to bend was restricted to any part of the proximal portion, although there was usually a decrease in the velocity or frequency of beating, and some changes in wave-form, which may be the results of mechanical constraints. The proximal portion continued to beat only if its length was at least $25-30 \%$ of the length of the entire tail, as shown in Text-fig. I. 
Visual observation of the spermatozoa was resumed within a second or two after irradiation. Beating had often stopped by this time, and when it did continue its frequency was usually much lower than before irradiation. The amplitude sometimes appeared normal, but was often greatly reduced. Beating often slowed gradually to a stop within a few seconds, although the proximal region of some spermatozoa beat sporadically for several seconds before stopping.

\section{Effects on the distal region of live spermatozoa}

As the photographs in Pl. I show, a variety of patterns of movement developed in the distal region after irradiation. The effects of irradiation exhibited several consistent features.

(i) Bends which were already present in the flagellum distal to the point of irradiation generally continued propagating to the tip after irradiation, rather than remaining in place or decaying as standing waves. This can be seen clearly in most of the photographs in Pl. $\mathrm{r}$.

(ii) Localized irradiation caused appreciable changes in the parameters of bent regions propagating along the distal portion. Most of these changes could not be correlated with any known experimental variables, and no parameter appeared to be necessarily conserved. These changes are described below.

(iii) New bends never developed distally to the point of irradiation. In Pl. I, figs. $3-5$ distal bent regions have passed completely down the tail by the final exposure, leaving the distal portion quiescent as the rest of the tail continues to beat. Distal regions failed to initiate bends even when the irradiated point was within a few $\mu \mathrm{m}$. of the head, as shown in Pl. I, figs. 7 and 8. The effects appear similar to those in flagella irradiated further back, except for an occasional spermatozoon such as that shown in Pl. I, fig. I I, in which a slight curvature developed behind the head. These curves did not seem to propagate, and were perhaps due to the forces of bends moving back along the flagellum.

(iv) Irradiation close to the trailing end of a bent region (the unbending point) did not prevent the propagation of that bent region, nor did it ever produce a situation in which the leading end of that bent region (the bending point) continued to propagate without being followed by an unbending point. An apparently complete bent region continued to propagate, as shown in Pl. 1 , figs. I, 2 and 6 . If the irradiation was farther into the bent region, that region was completely eliminated, as shown in Pl. I, figs. 5 and 10.

\section{Changes in the parameters of bends in the distal region}

The velocity of distal bent regions along the flagellum almost invariably decreased following irradiation. In approximately ro $\%$ of the injured spermatozoa this velocity quickly decreased to a new constant value of from about 30 to $90 \%$ of the original velocity. In Pl. I, figs. I and 2, for example, the velocities of bends in the distal region are about $\frac{2}{3}$ of the original values. In most of the spermatozoa the velocity of distal bends did not assume a constant value, but continued to decrease as the waves passed along the flagellum, as shown in Pl. I, fig. IO. In this case the velocity has decreased from about $40 \%$ of the original velocity between exposures $\mathrm{I}$ and 2 to about $15 \%$ between exposures 3 and 4 . 
The radius of curvature of bent regions in the distal portion did not decrease, and in approximately $25 \%$ of the injured spermatozoa it remained constant or nearly constant (within ro \% of its original value) after irradiation, as shown in Pl. I, figs. I and 6 . In approximately $10 \%$ of the cases the radius quickly increased to a new constant value, as shown in Pl. I, fig. 2. It was most common, however, for the radius to continue to increase as the bend passed along the flagellum, as shown in Pl. I, figs. 7 and 8. In some extreme cases, such as that shown in Pl. I, fig. 7, the radius was clearly not constant within a bent region, but increased toward the leading edge. Although exact measurement is difficult, the radius at a given point on the flagellum within a bent region appears to remain constant on two successive exposures, even though it is farther from the leading edge of that region in the latter exposure; that is, the radius at a given point appears to remain constant while that point is within a bent region. In cases where there were two bent regions in the distal portion the radius of the bend nearer the irradiated point sometimes appears to increase more than the more distant one. The greatest increases in radius of curvature were commonly associated with the largest decreases in propagation velocity.

An increase in the radius of curvature of a bent region was usually accompanied by an increase in length. This is shown particularly well in photographs such as Pl. I, fig. 8 , in which the shape of a bent region is retained as the radius increases. In some cases, however, the radius of a bent region increased without a corresponding increase in length, resulting in an increase in the angle $\alpha$ between the straight regions bordering that bent region, as shown in Pl. I, figs. 5 and 9 . There were no appreciable changes in $\alpha$ resulting from an increase in length without an increase in the radius, although this type of change can apparently occur in spermatozoa beating in solutions of abnormally high viscosity (Brokaw, 1966a).

\section{Effects of irradiation on glycerinated spermatozoa}

Glycerinated spermatozoa beat rather slowly and asymmetrically under the conditions necessary for irradiation, making stroboscopic determination of their frequency of beating very difficult, and exact determinations of beat parameters were not made. They are difficult to photograph, and only in a few experiments were they successfully photographed. The proximal region of glycerinated spermatozoa appeared to beat quite normally after irradiation, without obvious changes in wave parameters. In contrast to live spermatozoa, the proximal region of glycerinated ones commonly continued to beat for several minutes. As with live spermatozoa, bends already present in the distal portion of the flagellum continued to propagate to the tip, and no new bends were formed in the distal region, even when the proximal region continued to beat actively.

\section{DISCUSSION}

Damage to the flagellum was probably the result of intense local heating. Although the flagella appear to be sharply broken, the possibility that some effects may extend over a larger radius cannot be dismissed, and lack of exact knowledge of the effects on internal structures of course precludes a very detailed interpretation of these experiments in terms of flagellar ultrastructure. There are, however, some more general implications for the mechanism of flagellar beating. 
The inability of the distal region to beat, even when the irradiation was within a few $\mu \mathrm{m}$. of the basal end, suggests that the basal end is necessary for the initiation of bends While the mechanism of energy supply along the flagellum is unknown, and could possibly be diffusion of an energy-rich compound such as ATP from the basal end (Brokaw, 1966c; Raff \& Blum, 1968), the cessation of beating in the distal region does not seem to have been due simply to interruption of an ATP gradient along the tail. This is indicated by the following observations.

(i) Glycerinated flagella, in which the distal portion presumably received ATP from the surrounding solution, failed to initiate distal bends after irradiation.

(ii) In some cases intact flagella can be separated from their normal source of energy, and often remain vigorously motile for several seconds (Brokaw, 1965, and unpublished observations).

(iii) For a gradient to supply energy reliably for every beat, there must be an excess supply of ATP, so that at least a few beats would be expected to occur after irradiation, before the supply was exhausted. Similarly, calculations based on the volume and ATP usage of flagella, and reasonable estimates of intracellular ATP concentrations, suggest that flagella supplied with ATP by diffusion should contain enough ATP for many beats. However, no distal bends developed after irradiation.

Spermatozoa with tails broken by mechanical shearing (Brokaw, 1965) can swim vigorously, even if only a short region of the tail (perhaps 10\% of its length) remains (C. J. Brokaw, unpublished observations). The immediate cessation of beating in the proximal portion of flagella irradiated within $25 \%$ of the flagellar length from the basal end does not, therefore, appear to indicate a minimum length necessary for the initiation of bends. Rather, it may be the result of mechanical constraint caused by the irradiation and subsequent adhesion of the flagellum to the glass. This interpretation agrees well with the observations of Kaneda (1965) mentioned above, and might indicate constraints from normally sliding filaments which have been prevented from sliding at the irradiated point. Cilia and flagella have been removed from cells by several investigators, and can be made to beat in the presence of ATP, even though they have apparently been separated from their basal bodies. The capability for bend initiation therefore appears to be restricted to a short portion of a flagellum near its basal end.

Theories of the propagation of flagellar bending have been proposed by Machin $(1958,1963)$ and by Brokaw (1966b). According to Machin's theory, the flagellum contains a series of contractile elements arranged along its length. These elements tend to beat spontaneously, and are coupled to one another by the elastic properties of the flagellum. In the theory outlined by Brokaw, bends are initiated by an oscillator located near the base of the flagellum, and propagated by local events near the bending and unbending points. In the present experiments bending elements along the flagellum do not appear to be capable of independent beating, but appear to be triggered locally. However, bend propagation at a point was not affected solely by local activities: (i) the effects of irradiation on distal bends indicates that bending at a point can be immediately affected by activities in other parts of the flagellum (at least, by very drastic activities); (ii) the result of irradiation within a bent region suggests that bends are maintained as units, and bending was never propagated without being followed by unbending. 
Brokaw ( 1968 ) has recently discussed two alternative types of model for the generation of flagellar bends. The more familiar of these, referred to as a 'local contraction' model, achieves bending by means of contractile elements arranged along the flagellum, often presumed to be the outer fibrils of the axoneme. The second type of model, referred to as a 'sliding filament' model, achieves bending by longitudinal displacements rather than contractions. A flagellum is assumed to contain longitudinal filaments which are prevented from moving at the basal end. A bend is developed by sliding the filaments with respect to one another. In both types of model, cross-links between the active elements keep bending localized to small regions of the flagellum. Electron micrographs of the tip region of cilia by Satir (1964, 1967, 1968) are consistent with a sliding filament model involving the outer axonemal fibres as the active components, and indicate that, if bending is due to contraction, the outer axonemal fibres are not the contractile elements. Horridge $(1965)$ has reached somewhat similar conclusions.

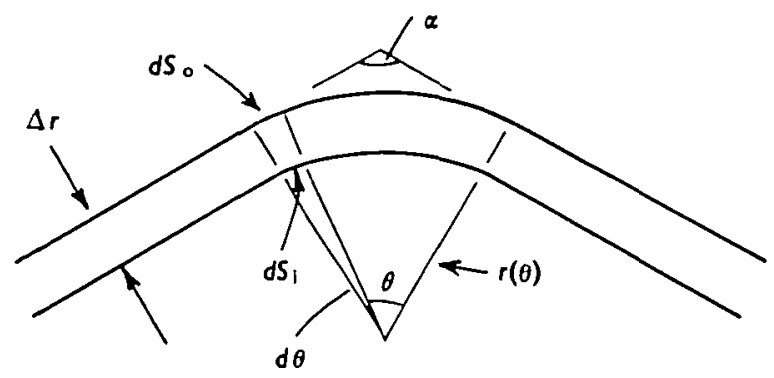

Tert-fig. 2. Parameters of a bent region. $\alpha$, angle between bordering straight regions; $\theta$, internal an gle of region; $r(\theta)$, radius of inner filament; $\Delta r$, distance between filaments; $S_{i}$, distance along inner filament; $S_{0}$ distance along outer filament.

The possibility of sliding filaments producing the effects observed in the proximal region of irradiated flagella has been mentioned above. In addition, sliding filaments might provide a means of producing the rapid changes seen in bends distal to the irradiated point. The development of new bends in a sliding filament model based on the outer axonemal fibres requires these fibres to slide along the entire length of the flagellum, since they do not appear to have slid past one another at their basal end in electron micrographs. Interruption of this movement at the irradiated point might easily result in immediate changes in bends distal to that point, although predicting the form these changes should take requires a more detailed theory than has been proposed.

However, the changes observed in the angle of bend $\alpha$ of distal bent regions is difficult to explain in terms of a sliding filament model. Text-fig. 2 illustrates some parameters of a bent region in a simplified diagram, in which only two filaments are shown: one on the concave side of a bent region (inside) and one on the convex side (outside). If the filaments are assumed to be incompressible and inextensible, and separated by a constant distance $\Delta r$, then an increment of length along the inner filament is

$$
d S_{i}(\theta)=r(\theta) d \theta
$$

Similarly,

$$
d S_{0}(\theta)=[r(\theta)+\Delta r] d \theta
$$


The incremental difference in the lengths of the filaments is

$$
d S(\theta)=d S_{0}(\theta)-d S_{i}(\theta)=\Delta r d \theta
$$

the total difference in the lengths of the filaments within the bent region is

So

$$
\Delta S=\int_{\theta} d S(\theta)=\theta \Delta r=(\pi-\alpha) \Delta r .
$$

$\alpha=\pi-\Delta S / \Delta r$

independently of the shape of that region or the mechanism of bending; the total angle $\alpha$ of a bent region is determined solely by the difference in the lengths of the filaments on the convex and concave sides of that region. In a flagellum containing several such bent regions, the angle between the ends of the flagellum is determined by the net sliding of the filaments. The observation of Brokaw ( $196_{5}$ ) that thioureatreated spermatozoa maintain a constant angle between the head and the tip of the tail further suggests constraints imposed by a sliding filament system. However, the total angle of distal bent regions of irradiated flagella often increased appreciably, without preventing those regions from propagating. While an increase in $\alpha$ merely requires the elements of a contractile model to contract less than normally, it requires the filaments of a sliding filament model to slide past one another. The latter model thus has the difficult task of maintaining and propagating a bent region by cross-links between the filaments while the filaments are sliding and breaking these cross-links, in a manner quite different from the normal beat pattern. A related difficulty has been mentioned by Gibbons \& Grimstone ( 1960$)$.

While some of the results suggest constraints imposed by sliding filaments, others seem more easily explained on the assumption that the forces producing bending involve some form of contraction. The variety of effects does not appear easily explainable by any of the existing models of flagellar bending.

\section{SU M M A R Y}

I. Sea-urchin and starfish sperm tails were irradiated at pre-selected points along the flagellum and at pre-selected phases of the beat cycle by means of a pulsed ruby laser microbeam. Multiple-exposure dark-field photomicrographs were taken immediately before and after irradiation. The flagellum usually appeared to be broken at the irradiated point.

2. The portion of a flagellum between the head and the irradiated point continued for at least a few beats if its length was at least $25 \%$ of the length of the tail, and stopped immediately if it was shorter.

3. Bends already established beyond the irradiated point continued to propagate to the tip of the flagellum. Their propagation velocity generally decreased, and there were usually changes in other bend parameters.

4. No new bends ever developed beyond the irradiated point.

5. Irradiation within a bent region often completely eliminated that region.

I am indebted to Professor C. J. Brokaw for guidance during the course of these experiments, and for generous criticism of the first draft of this paper. This work was supported by U.S. Public Health Service Training Grant 5 TI GM 86 and U.S. Public Health Service Grants GM 6965 and GM 14613. 


\section{REFERENCES}

AfZelius, B. A (1955). The fine structure of the sea urchin spermatozoon as revealed by the electron microscope. Z. Zellforsch. mikrosk. Anat. 42, 134-48.

BAkKR, C. L. (1963). Spermatozoa and spermateliosis in Cryptobranchus and Necturis. 9. Tenn. Acad. Sci. $\mathbf{3}^{8}, \mathrm{r}-\mathbf{9}$.

Brokaw, C. J. (I961). Movement and nucleoside polyphosphatase actavity of 1solated flagella from Polytoma revella. Expl Cell Res. 22, $151-62$.

Brokaw, C. J. (1963). Movement of the flagella of Polytoma uvella. F. exp. Biol. 40, 149-56.

Brokaw, C. J. (1965). Non-8inusoidal bending waves of sperm flagella. J. exp. Brol. 43, i55-69.

BrokAw, C. J. (1966a). Effects of increased viscosity on the movements of some invertebrate spermatozoa. 7. exp. Bzol. 45, I13-39.

BrokAw, C. J. (1966b). Bend propagation along flagella. Nature, Lond. 209, 161-3.

Brokaw, C. J. (I966c). Mechanics and energetics of cilia. Am. Rev, resp. Dis. 93, 32-40.

Brokaw, C. J. (1968). Mechanısm of sperm movement. Symp. Soc. exp. Biol. 22, 101-116.

Brokaw, C. J. \& BENEDICT, B. (1968). Mechano-chemical coupling in flagella. I. Movement-dependent dephosphorylation of ATP by glycerinated spermatozoa. Archs Biochem. Biophys. 125, 770-8.

Colour Index, second ed.(1956). Bradford, Yorkshire, England: Society of Dyers and Colourists ; Lowell, Mass., U.S.A.: American Association of Textile Chemists and Colourssts, Lowell Technical Instutute.

Engermann, T. W. (1898). Cils Vibratils. In Dictionnatrede Physiologie. Ed. C. Richet. Paris: F. Alcan.

Gibbons, I. R. \& Grimstone, A. V. (1960). On flagellar structure in certain flagellates. Y. biophys. biochem. Cytol. 7, 697-716.

Goldstein, S. F. (1968). Ph.D. Thesis. Calıfornia Institute of Technology.

Gray, J. (1955). The movement of sea-urchin spermatozoa. F. exp. Biol. 32, 775-801.

Gray, J. (1958). The movement of spermatozoa of the bull. F. exp. Biol. 35, 96-1 08 .

Hoffmann-Berling, H. (I955). Geisselmodelle und Adenosintryphosphat (ATP). Biochim. brophys. Acta 16, 146-54.

Holwill, M. E. J. (1965). The motion of Strigomonas oncopelti. F. exp. Biol. 42, 125-37.

HorRidGe, G. A. (1965). Macrocilia with numerous shafts from the lips of the ctenophore Beroe. Proc. R. Soc. B I62, $35 \mathrm{I}-64$.

KankDA, Y. (1965). Movement of sperm tail of frog J. Fac. Sci. Tokyo Univ. (Sect. IV) ro (part 3), 427-40.

LENGYeL, B. A. (1962). Lasers, p. 55. New York: J. Wiley and Sons.

Machin, K. E. (1958). Wave propagation along flagella. Y. exp. Biol. 35, 796-806.

Machin, K. E. (r963). The control and synchronisation of flagellar movement. Proc. $R$. Soc. B 158, 88-104.

Nelson, L. (1959). Cytochemical studies with the electron microscope. II. Succinic dehydrogenase in rat spermatozoa. Expl Cell Res. 16, 403-10.

NeISON, L. (1962). Cytochemical aspects of spermatozoan motulity. In Spermatozoan Motility, pp. I7I-87. Ed. D. W. Bishop. Washington, D.C.: American Association for the Advancement of Science.

RAFF, E. C. \& Blum, J. J. (I 968). A possible role for adenylate kinase in calia: concentration profiles in a geometrically constrained system. F. theor. Biol. 18, 53-71.

SAKs, N. M., ZuzoLo, R. C. \& KopAC, M. J. (1965). Microsurgery of living cells by ruby laser irradıation. In The Laser, Ann. N.Y. Acad. Sct. 122, 695-712.

SatIR, P. (1964). Filament-matrix interaction during ciliary movement: inferences drawn from electron microscopy of the distal end of the cliary shaft of lamellibranch gll cilia. 9. Cell Biol. 23, 82 A.

Satir, P. (I967). Morphological aspects of ciliary motility. F. gen. Physiol. 50, $241-58$.

SatrR, P. (rg68). Studies on calia III. Further studies on the cilium tip and a 'sliding filament' model of ciliary motility. 7. Cell Biol. 39, 77-94.

Terni, T. (1933). Microdissection et U.V. microradiopiqure des spermatozoides. C. r. Ass. Anat. 28, $651-54$.

TYLER, A. (1949). A simple, non-injurious, method for inducing repeated spawning of sea urchins and sand dollars. Collecting Net r9, 19-20.

Trukr, A. (1953). Prolongation of life-span of sea urchin spermatozoa, and improvement of the fertilization-reaction, by treatment of spermatozoa and eggs with metal-chelating agents (amino acids, Versene, DEDTC, oxine, cupron). Biol. Bull. mar. biol. Lab., Woods Hole 104, 224-39.

W Alker, P. J. (1961). Organisation of function in Trypanosome Flagella. Nature, Lond. 189, 101 7-18. 


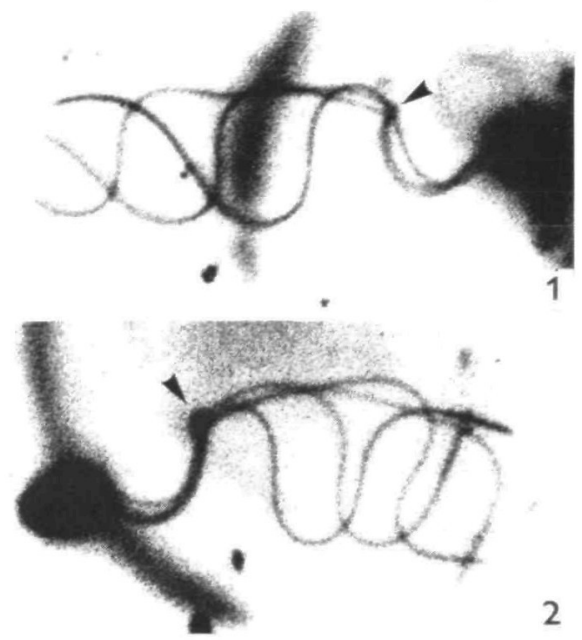

2
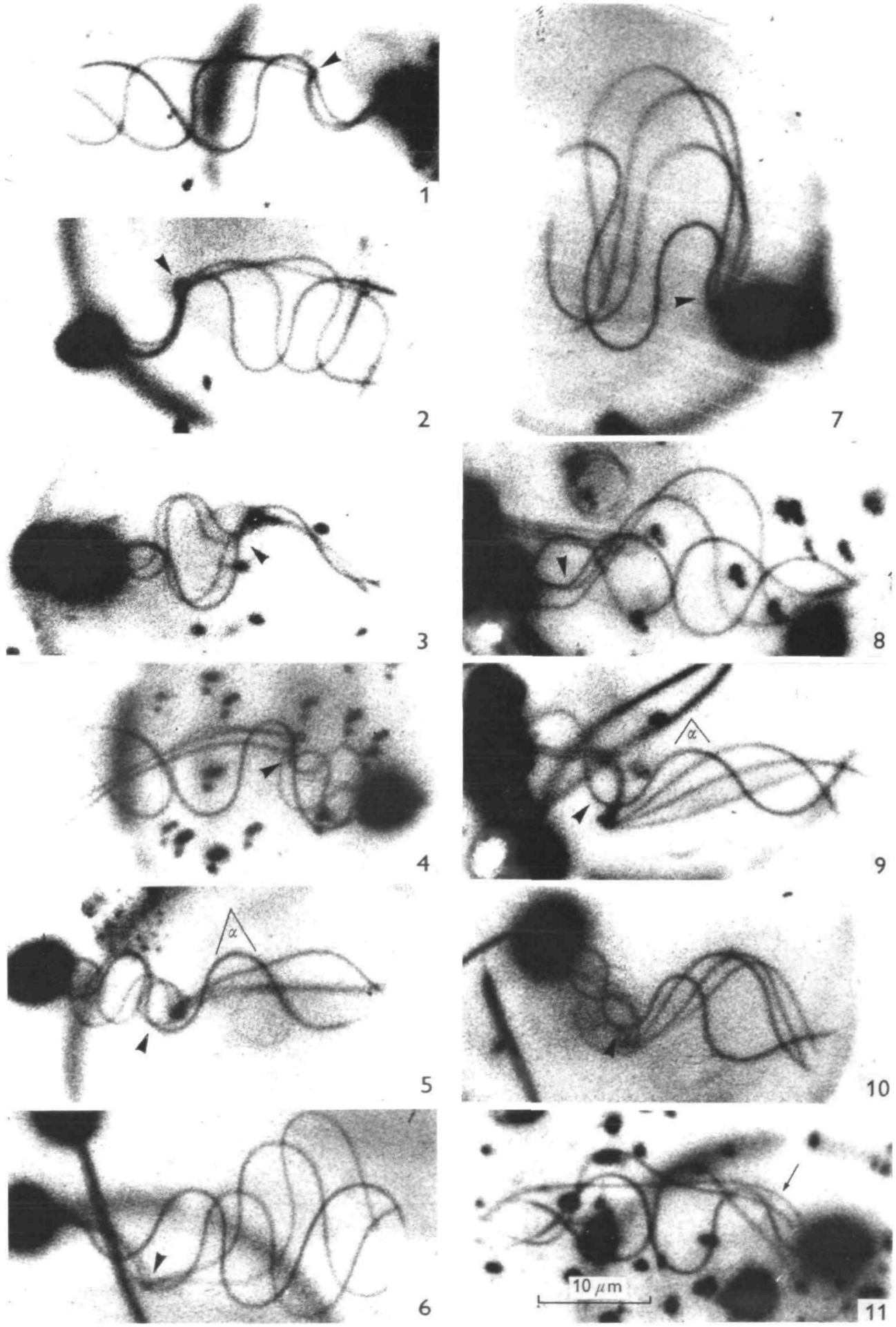

STUART F GOLDSTEIN

(Facing $p+41$ ) 


\section{EXPLANATION OF PLATE}

All the photographs are of spermatozoa of Strongylocentrotus purpuratus except figs. 6 and 7, which are of spermatozoa of Pisaster brevuspinus. In figs. 6 and 8 two exposures have been taken before irradiation. $\alpha$ denotes total angle of a bent region. Arrowhead indicates irradiated point. Arrow in fig. I I indicates a curve developed after irradiation. Frequencies of beating before irradation were:

$\begin{array}{cccccc}\text { Fig. } & \mathrm{Hz} & \text { Fig. } & \mathrm{Hz} & \text { Fig. } & \mathrm{Hz} \\ \text { I } & 18 \cdot 3 & 5 & 23 \cdot 2 & 9 & 24 \cdot 0 \\ 2 & 20 \cdot 0 & 6 & 20 \cdot 3 & 10 & 26 \cdot 8 \\ 3 & 26 \cdot 0 & 7 & 16 \cdot 7 & 11 & 24 \cdot 8 \\ 4 & 25 \cdot 7 & 8 & 20 \cdot 2 & & \end{array}$

